\title{
Usefulness of magnetic resonance imaging-guided vacuum-assisted breast biopsy in Korean women: a pilot study
}

Yeong Yi An ${ }^{1}$, Sung Hun Kim², Bong Joo Kang ${ }^{2^{*}}$ and Jae Hee Lee ${ }^{3}$

\begin{abstract}
Background: Magnetic resonance imaging (MRI)-guided vacuum-assisted biopsy is the technique of choice for lesions that are visible only with breast MRI. The purpose of this study was to report our clinical experience with MRI-guided vacuum-assisted biopsy in Korean women.
\end{abstract}

Methods: A total of 13 patients with 15 lesions for MRl-guided vacuum-assisted biopsy were prospectively entered into this study between September 2009 and November 2011. Biopsy samples were obtained in a 3-T magnet using a 9-guage MRI-compatible vacuum-assisted biopsy device. We evaluated clinical indications for biopsy, lesion characteristics on prebiopsy MRI, pathologic results, and postbiopsy complication status.

Results: The clinical indications for MRI-guided vacuum-assisted biopsy were as follows: abnormalities in patients with interstitial mammoplasty on screening MRI $(n=10)$; preoperative evaluation of patients with a recently diagnosed cancer $(n=3)$; and suspicious recurrence on follow-up MRI after cancer surgery $(n=1)$ or chemotherapy $(n=1)$. All lesions have morphologic features suspicious or highly suggestive of malignancy by the American College of Radiology Breast Imaging Reporting and Data System category of MRI (C4a $=12, C 4 b=2, C 5=1)$. In two of the 15 lesions (13.3\%, <6 mm), MRl-guided 9-gauge vacuum-assisted breast biopsy was deferred due to nonvisualization of the MRI findings that led to biopsy and the lesions were stable or disappeared on follow up so were considered benign. Of 13 biopsied lesions, pathology revealed four malignancies (4/13, 30.8\%; mean size $15.5 \mathrm{~mm}$ ) and nine benign lesions (9/13, 69.2\%; size $14.2 \mathrm{~mm}$ ). Immediate postprocedural hematoma (mean size $23.5 \mathrm{~mm}$ ) was observed in eight out of 13 patients (61.5\%) and was controlled conservatively.

Conclusions: Our initial experience of MRI-guided vacuum-assisted biopsy showed a success rate of $86.7 \%$ and a cancer diagnosis rate of $30.8 \%$, which was quite satisfactory. MRI-guided vacuum-assisted breast biopsy is a safe and effective tool for the workup of suspicious lesions seen on breast MRI alone without major complication. This biopsy may contribute to the early diagnosis of breast cancer in interstitial mammoplasty patients in Korea.

\section{Background}

Magnetic resonance imaging (MRI) of the breast is the most sensitive technique for the detection, diagnosis, and treatment planning of breast cancer with a high sensitivity of 90 to $99 \%$. Although MRI has a high sensitivity in detecting breast lesions, its specificity is comparatively low, ranging from 37 to $72 \%$ [1]. Because of the limited specificity of MRI, histopathological verification is required

\footnotetext{
* Correspondence: lionmain@catholic.ac.kr

${ }^{2}$ Department of Radiology, Seoul St. Mary's Hospital, College of Medicine, The Catholic University of Korea, 505 Banpo-Dong, Seocho-Ku, Seoul 137-040, Republic of Korea

Full list of author information is available at the end of the article
}

to avoid unnecessary surgical biopsies of benign lesions detected by MRI.

The increasing use of MRI yields a number of MRIonly visible suspicious lesions that cannot be identified by mammography or ultrasonography. A MRI-guided tissue sampling method (MRI-guided percutaneous core biopsy or vacuum-assisted biopsy) is the technique of choice for lesions that are visible only on breast MRI, and it has been performed successfully for many years in the western countries [2-12]. In contrast, second-look ultrasound and ultrasound-guided tissue sampling is usually performed in Korea, and MRI-guided tissue sampling is only performed at a few university hospitals.

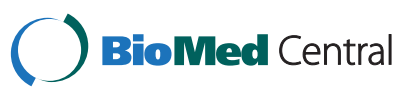


Except for the recently published preliminary experience with MRI-guided vacuum-assisted breast biopsy (VABB) in Japan [13-15], there are no published data in Asian or Korean women. The aim of this prospective study was therefore to evaluate our initial clinical experience with MRI-guided VABB of breast lesions visible only on MRI in Korean women. We report our initial clinical experience in 15 cases scheduled for MRI-guided 9-gauge VABB of MRI-detected lesions interpreted as suspicious or highly suggestive of malignancy.

\section{Methods}

\section{Patient population}

This was a prospective study for assessing the usefulness of MRI-guided VABB, approved by the Institutional Review Board of our hospital. Informed consent was obtained from all patients. A total of 13 patients with 15 lesions scheduled for MRI-guided VABB and surgical excision between September 2009 and November 2011 were asked to participate in this prospective study. The patients ranged in age from 35 to 73 years and the median age was 51.4 years.

\section{Breast MRI technique and lesion characteristics before biopsy}

The MRI scans were acquired with the patient in the prone position with a $1.5 \mathrm{~T}$ scanner (Achieva; Philips Medical Systems, Best, the Netherlands) equipped with a breast coil.

The MRI images with the Achieva scanner were acquired using the following sequences: sagittal, fatsuppressed, and fast spin-echo T2-weighted imaging sequence (Repetition time/echo time 6,000/100 ms, flip angle $90^{\circ}, 30$ slices, field of view of $320 \mathrm{~mm}$, matrix $424 \times$ 296 , number of excitations of $1,4 \mathrm{~mm}$ slice thickness with

Table 1 Clinical indications, lesion characteristics on prebiopsy magnetic resonance imaging, and pathologic diagnosis in 15 lesions

\begin{tabular}{|c|c|c|c|c|c|c|c|c|}
\hline \multirow[t]{2}{*}{ Number } & \multirow[t]{2}{*}{ Indications } & \multicolumn{5}{|c|}{ Lesion characteristics on MRI } & \multicolumn{2}{|l|}{ Pathology } \\
\hline & & Type & $\begin{array}{l}\text { Size } \\
(\mathrm{mm})\end{array}$ & Findings & Kinetics & $\begin{array}{l}\text { BI-RADS } \\
\text { category }\end{array}$ & $\begin{array}{l}\text { Confirmative } \\
\text { methods }\end{array}$ & $\begin{array}{l}\text { Pathologic } \\
\text { diagnosis }\end{array}$ \\
\hline 1 & Interstitial mammoplasty, screening & Mass & 22 & $\begin{array}{l}\text { Lobular, smooth, } \\
\text { rim }\end{array}$ & Fast/washout & $c 4 b$ & $\begin{array}{l}\text { VABB/ } \\
\text { surgery }\end{array}$ & IDC \\
\hline 2 & Interstitial mammoplasty, screening & Mass & 14 & $\begin{array}{l}\text { Lobular, smooth, } \\
\text { homogeneous }\end{array}$ & Fast/plateau & $\mathrm{C} 4 \mathrm{a}$ & $\begin{array}{l}\text { VABB/ } \\
\text { surgery }\end{array}$ & IDC \\
\hline 3 & $\begin{array}{l}\text { Interstitial mammoplasty, ipsilateral } \\
\text { breast cancer, chemotherapy follow-up }\end{array}$ & Mass & 8 & $\begin{array}{l}\text { Irregular, irregular, } \\
\text { homogeneous }\end{array}$ & Fast/washout & $c 4 b$ & VABB & IDC \\
\hline 4 & Interstitial mammoplasty, screening & Mass & 18 & $\begin{array}{l}\text { Irregular, spiculated, } \\
\text { heterogeneous }\end{array}$ & Fast/washout & C5 & $\begin{array}{l}\text { VABB/ } \\
\text { surgery }\end{array}$ & DCIS \\
\hline 5 & Interstitial mammoplasty, screening & Mass & 26 & $\begin{array}{l}\text { Lobular, irregular, } \\
\text { heterogeneous }\end{array}$ & Fast/plateau & $\mathrm{C} 4 \mathrm{a}$ & VABB & FA \\
\hline 6 & Interstitial mammoplasty, screening & Mass & 8 & $\begin{array}{l}\text { Irregular, irregular, } \\
\text { homogeneous }\end{array}$ & Fast/plateau & C4a & VABB & FA \\
\hline 7 & Interstitial mammoplasty, screening & Mass & 5 & $\begin{array}{l}\text { Round, irregular, } \\
\text { homogeneous }\end{array}$ & Fast/plateau & $\mathrm{C} 4 \mathrm{a}$ & VABB & FA \\
\hline 8 & Interstitial mammoplasty, screening & Mass & 28 & $\begin{array}{l}\text { Lobular, irregular, } \\
\text { heterogeneous }\end{array}$ & $\begin{array}{l}\text { Medium/ } \\
\text { persistent }\end{array}$ & $\mathrm{C} 4 \mathrm{a}$ & VABB & FCC \\
\hline 9 & Interstitial mammoplasty, screening & Mass & 16 & $\begin{array}{l}\text { Oval, irregular, } \\
\text { heterogeneous }\end{array}$ & Fast/plateau & $\mathrm{C} 4 \mathrm{a}$ & VABB & FCC \\
\hline 10 & Interstitial mammoplasty, screening & Mass & 6 & $\begin{array}{l}\text { Oval, irregular, } \\
\text { homogeneous }\end{array}$ & Fast/washout & $\mathrm{C} 4 \mathrm{a}$ & VABB & FCC \\
\hline 13 & Interstitial mammoplasty, screening & Mass & 9 & $\begin{array}{l}\text { Oval, smooth, } \\
\text { heterogeneous }\end{array}$ & Fast/washout & $\mathrm{C} 4 \mathrm{a}$ & VABB & $\begin{array}{l}\text { Silicone } \\
\text { mastitis }\end{array}$ \\
\hline 11 & Breast cancer, preoperative staging & Mass & 8 & $\begin{array}{l}\text { Oval, smooth, } \\
\text { heterogeneous }\end{array}$ & $\begin{array}{l}\text { Medium/ } \\
\text { plateau }\end{array}$ & $\mathrm{C} 4 \mathrm{a}$ & VABB & FCC \\
\hline 12 & Breast cancer follow-up & Nonmass & 11 & Focal, clumped & $\begin{array}{l}\text { Medium/ } \\
\text { plateau }\end{array}$ & $\mathrm{C} 4 \mathrm{a}$ & VABB & FCC \\
\hline 14 & Breast cancer, preoperative staging & Mass & 6 & $\begin{array}{l}\text { Oval, smooth, } \\
\text { heterogeneous }\end{array}$ & Fast/washout & $\mathrm{C} 4 \mathrm{a}$ & MRI FU & NA \\
\hline 15 & Breast cancer, preoperative staging & Nonmass & 5 & $\begin{array}{l}\text { Segmental, } \\
\text { heterogeneous }\end{array}$ & $\begin{array}{l}\text { Medium/ } \\
\text { plateau }\end{array}$ & $\mathrm{C} 4 \mathrm{a}$ & MRI FU & NA \\
\hline
\end{tabular}


$0.1 \mathrm{~mm}$ interslice gaps, and acquisition time of 2 minutes 56 seconds) and precontrast and postcontrast dynamic axial T1-weighted three-dimensional, fat-suppressed, fatspoiled gradient-echo sequence (Repetition time/echo time 6.9/3.4, flip angle of $12^{\circ}, 2.0 \mathrm{~mm}$ slice thickness with no gap, acquisition time of 1 minute 31 seconds). The images were obtained before and at $0,91,182,273,364$, and 455 seconds after a rapid bolus injection of gadoliniumdiethylenetriamine pentaacetic acid (Magnevist; Schering, Berlin, Germany) at $0.1 \mathrm{mmol} / \mathrm{kg}$ of body weight.

Two breast radiologists analyzed the imaging findings according to the American College of Radiology Breast Imaging Reporting and Data System categories for MRI [16]. All lesions were classified into category 4 or category 5 on MRI. For all cases, the following lesion characteristics were recorded: type (focus, mass or nonmass enhancement), size, location, and kinetic analysis.

\section{MRI-guided vacuum-assisted breast biopsy procedure}

Biopsies were performed with a 9-gauge MRI-compatible vacuum-assisted biopsy device (Automated Tissue Excision and Collection; Suros Surgical Systems, Indianapolis, IN, U.S). All biopsies were performed by two radiologists specializing in breast imaging (BJK, SHK); all had previous experience in breast MRI and percutaneous biopsies under stereotactic and sonographic guidance. The two radiologists had not previously performed the procedure but had observed or assisted one or more of the experienced radiologists.

The MRI biopsy technique has been previously described in detail [9]. The patient was positioned prone in the $1.5 \mathrm{~T}$ magnet (Signa, GE Medical Systems, Milwaukee, WI, USA). The affected breast was placed in a dedicated biopsy compression device using a commercially available grid localizing system. The protocol for MRI-guided VABB

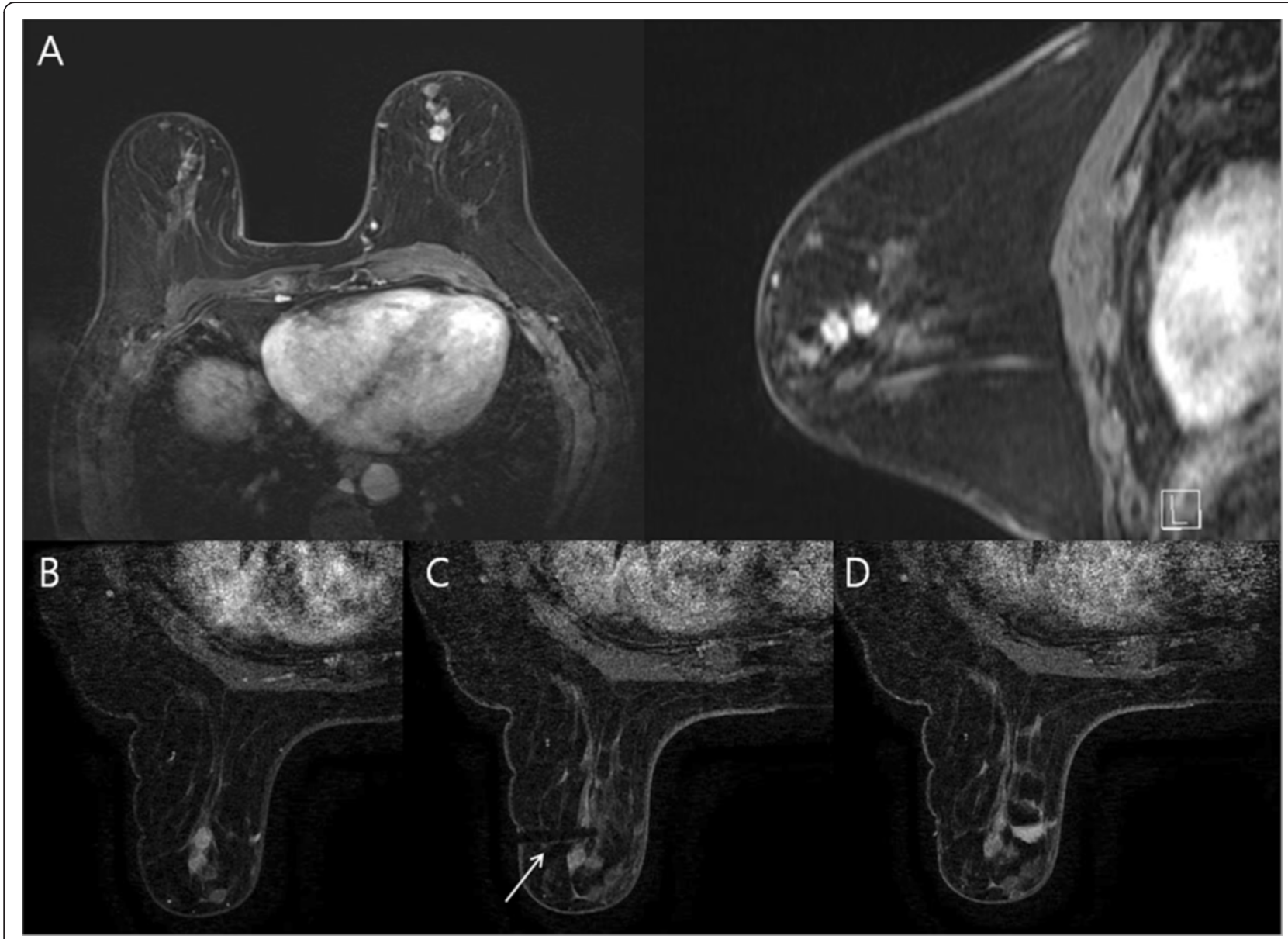

Figure 1 Example of invasive ductal carcinoma after silicone injection in the breast. A 73-year-old woman who received silicone injection had a suspicious lesion in the left breast on magnetic resonance imaging (MRI) screening. (A) Axial and sagittal contrast enhanced T1-weighted image showing clustered, lobular, rim-enhancing masses with relatively smooth margins in the left breast, which were categorized into American College of Radiology Breast Imaging Reporting and Data System category 4b. (B) Axial T1-weighted subtracted dynamic image acquired immediately before biopsy showing clustered enhancing masses. (C) Axial T1-weighted subtracted dynamic image of the left breast showing the presence of the obturator (arrow) within the mass. (D) Axial T1-weighted subtracted dynamic image of the left breast immediately after 9-gauge MRI-guided vacuum-assisted biopsy showing air and hematoma at the biopsy site. Histologic analysis demonstrated invasive ductal carcinoma. 
at our institution comprised the following six steps: step 1, targeting images (breast compression by the biopsy guidance grid, marking the expected lesion site with a fiducial marker on the skin, and a sagittal T1 fat-saturated threedimensional spoiled gradient echo image before and after intravenous administration of $20 \mathrm{ml}$ gadodiamide (Omniscan; GE Healthcare, Oslo, Norway) plus $10 \mathrm{ml}$ saline); step 2, determining lesion location and desired depth of probe insertion; step 3, preparing the probe; step 4, placing the device, and performing imaging to confirm the lesion location (sterilization and anesthetization of the breast, placement of the introducer and stylet through the needle guide, removal of the stylet and placement of the obturator inside the introducer, and axial scan to confirm accurate targeting); step 5, performing the biopsy, postexamination images and collecting the specimens (removal of the obturator and insertion of the biopsy device, tissue sampling, and post-biopsy sagittal scan to assess the completeness of tissue acquisition and to examine for the presence of postprocedural complications); and step 6, post-biopsy care of the breast (compression with ice and application of a sterile dressing on the biopsy site).

\section{Data analysis}

Data collection - including clinical indications for breast MRI, patient age, menopausal status, lesion size and type on MRI, biopsy parameters, histologic results of vacuumassisted biopsy and surgical excision, and complications was performed by reviewing medical records. The time of the biopsy (minutes) was determined by calculating the interval between the beginning of the MRI localizing sequence and the end of the final MRI sequence acquired. The radiologist performing the biopsy reviewed the MRIs obtained during and after the biopsy to determine the

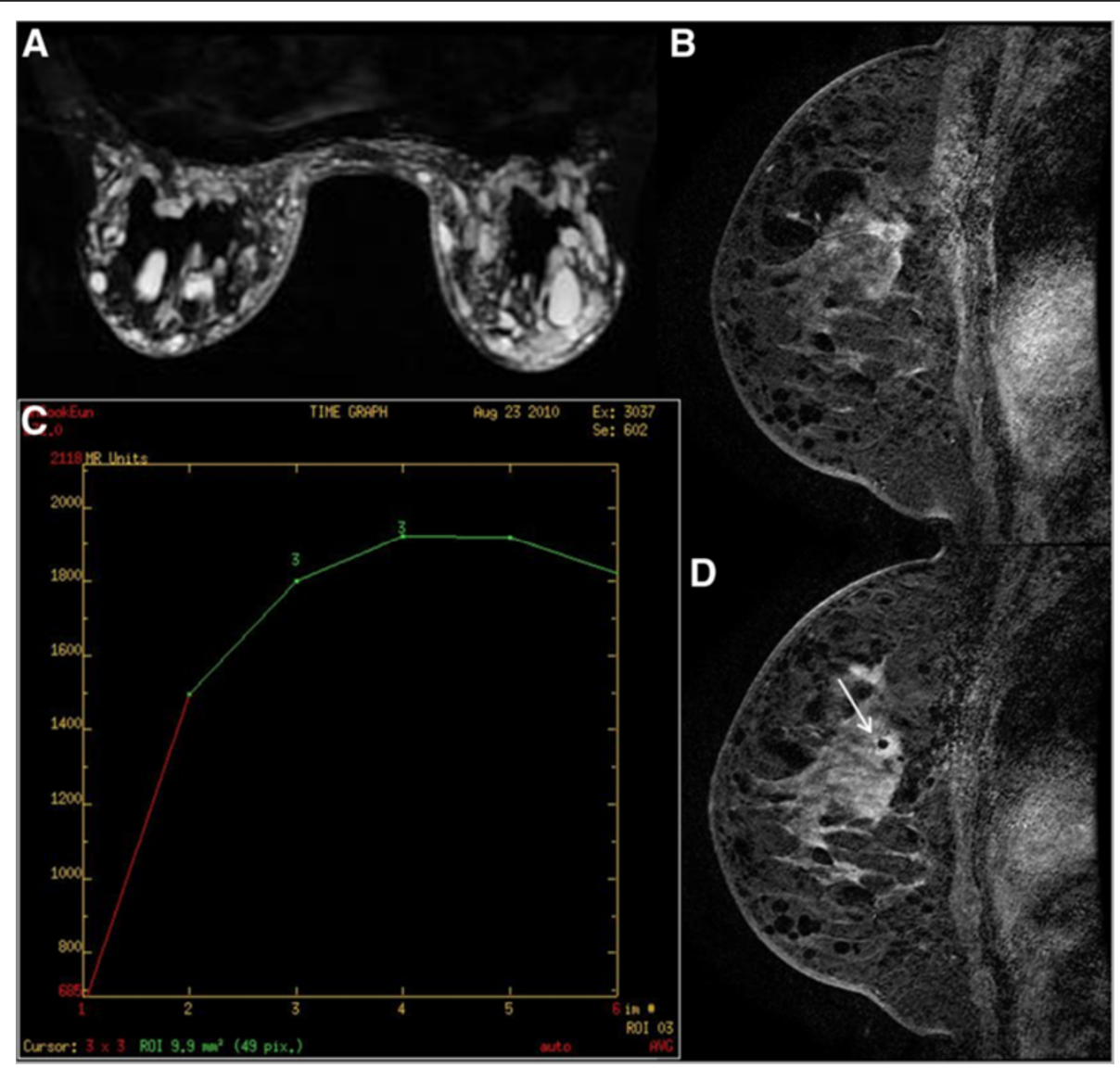

Figure 2 Example of ductal carcinoma in situ after silicone injection in the breast. A 44-year-old woman who received silicone injection had a suspicious lesion in the left breast on magnetic resonance imaging (MRI) screening. (A) Silicone granulomas showing high signal intensities on the inversion recovery MRI with chemically selective water suppression. (B) Sagittal T1-weighted subtracted dynamic image of the left breast showing irregular, speculated, heterogeneous enhancing mass, which was categorized into American College of Radiology Breast Imaging Reporting and Data System category 5. (C) Dynamic time-intensity curves of the lesion showing an initial fast uptake followed by a delayed washout (type III). (D) Sagittal T1-weighted subtracted dynamic image of the left breast showing the obturator tip (arrow) within the mass. Histologic analysis demonstrated ductal carcinoma in situ. 
presence and extent of postbiopsy changes, to assess whether the MRI target was sampled or possibly excised.

\section{Results}

\section{Clinical indications for MRI-guided vacuum-assisted breast biopsy}

The clinical indications for MRI-guided VABB were as follows: abnormalities in patients with interstitial mammoplasty on screening MRI $(n=10)$; presurgical evaluation of patients who were recently diagnosed with cancer $(n=3)$; and suspected recurrence on follow-up MRI after cancer surgery $(n=1)$ or chemotherapy $(n=1)$. None of the 15 lesions that were seen on MRI were detected by mammography, diagnostic or second-look ultrasonography.

\section{Lesion characteristics on pre-biopsy MRI}

The MRI-detected lesions included 13 masses and two nonmass enhancements. The mean diameter of lesions on prebiopsy MRI was $1.27 \mathrm{~mm}$ (range, 5 to $28 \mathrm{~mm}$ ). The clinical indications and MRI features with kinetic analysis of 15 lesions are summarized in Table 1. All lesions had morphologic features suspicious or highly suggestive of malignancy according to the American College of Radiology Breast Imaging Reporting and Data System category of MRI $(\mathrm{C} 4 \mathrm{a}=12, \mathrm{C} 4 \mathrm{~b}=2, \mathrm{C} 5=1)$.

\section{Biopsy procedure and histopathologic results}

MRI-guided VABB was successfully performed in 13 of 15 lesions (86.7\%). In two lesions (13.33\%), MRI-guided VABB could not be performed because the lesions disappeared on the scheduled day of biopsy. The mean procedural time was 55.7 minutes (range 20 to $90 \mathrm{mi}$ nutes). An average number of nine specimens were obtained per lesion (range, 6 to 11 ).

Histopathological results of the VABB and findings of the surgical specimens are summarized in Table 1. Of the 13 lesions sampled, four lesions were malignant $(30.8 \%$,

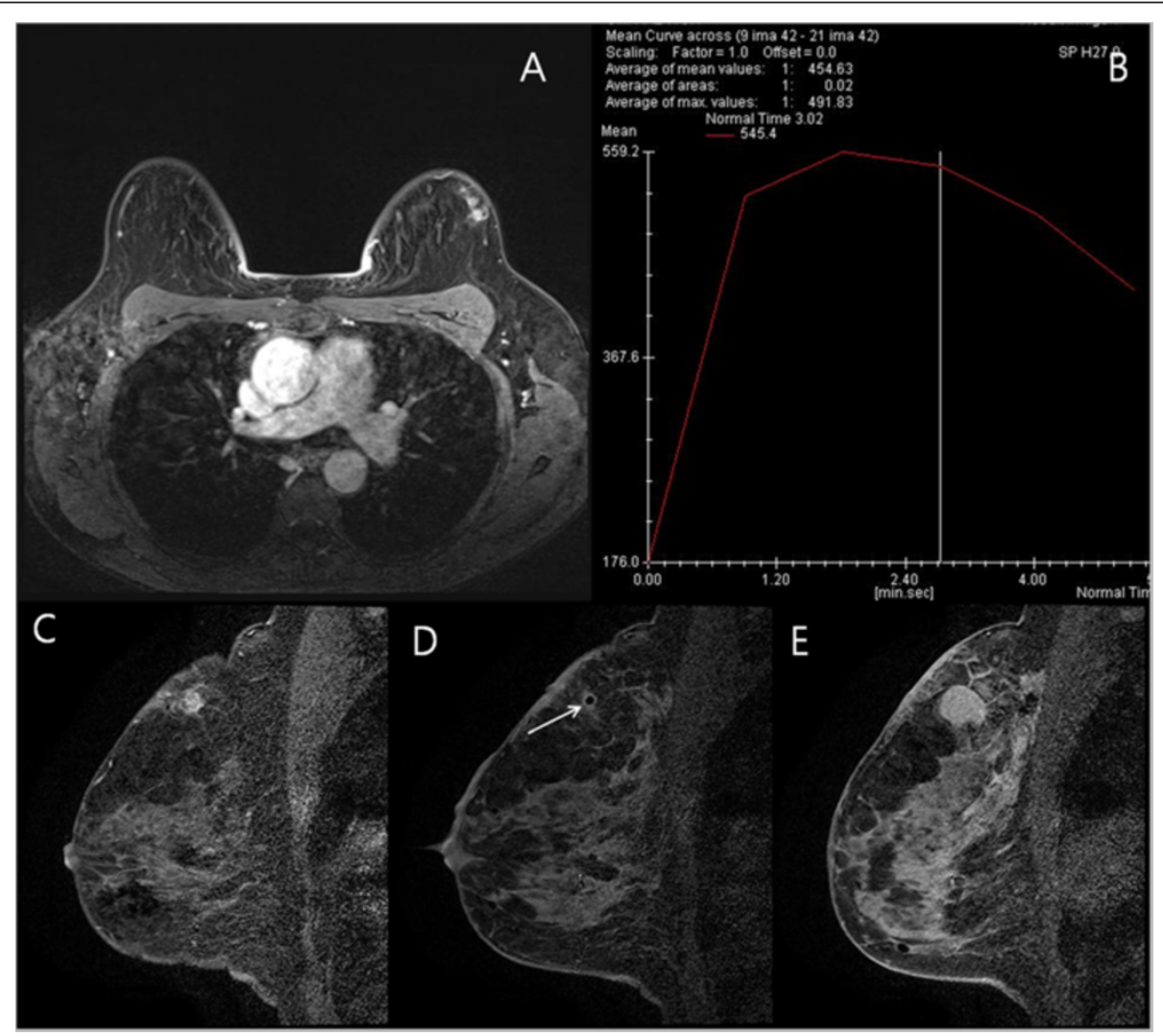

Figure 3 Example of silicone mastitis after silicone injection in the breast. A 47-year-old woman who received silicone injection had a suspicious lesion in the left breast on magnetic resonance imaging (MRI) screening. (A) Axial contrast enhanced T1-weighted image showing oval, smooth, heterogeneous enhancing mass in the left breast, which was categorized into American College of Radiology Breast Imaging Reporting and Data System category 4a. (B) Dynamic time-intensity curves of the lesion showing an initial fast uptake followed by a fast washout in the delayed phase (type III). (C) Sagittal, T1-weighted subtracted dynamic image acquired immediately before biopsy showing heterogeneously enhancing mass in the 12:00 hours position. (D) Sagittal T1-weighted subtracted dynamic image acquired after placement of the obturator showing low signal within the lesion (arrow), indicating the location of the obturator tip. (E) Sagittal T1-weighted subtracted dynamic image immediately after completion of tissue acquisition showing hematoma formation at the biopsy site. Histologic analysis demonstrated silicone mastitis. 
4/13) (Figure 1 and Figure 2) and the remaining nine lesions were benign $(69.2 \%, 9 / 13)$ (Figure 3).

Among the cases of confirmed malignancies, three lesions were invasive ductal carcinoma (Figure 1) and one was ductal carcinoma in situ (Figure 2). All cancers diagnosed by VABB were the mass type of enhancement. Malignant lesions diagnosed by VABB were surgically excised in three out of four cases. One patient did not undergo surgery due to bone metastasis detected by bone scan and positron emission tomography-computed tomography. Residual cancer was found at surgery in two out of three patients. One patient, in whom the MRI target was considered to have been completely excised, had no residual lesion. All benign diagnoses were confirmed by imaging-histologic correlation and by follow-up MRI at 6 to 12 months after biopsy. There were no missed cancers in the patients having benign lesions with concordant histology on MRI-guided biopsy.

\section{Lesions with deferred biopsy}

There were two lesions in which the biopsy was deferred. The lesion type was foci measuring $4 \mathrm{~mm}$ and a small mass measuring $6 \mathrm{~mm}$ on the initial MRI (Figure 4). Follow-up MRI within 6 months revealed resolution or decrease in the size of the lesions.

\section{Postbiopsy complications}

MRI performed immediately after biopsy showed that complete removal of the target lesion was achieved in one patient (7.7\%), partial removal of the target lesion was achieved in seven patients (53.8\%), and there was difficulty in assessing the removal of the target lesion due to bleeding in five patients (38.5\%).

Postprocedural hematoma formation was observed in eight patients (61.5\%), and was controlled conservatively. The mean size of the hematoma was $23.5 \mathrm{~mm}$ (range, 21 to $28 \mathrm{~mm}$ ). Postprocedural defect was observed in three patients and the mean size of the defect was $14.3 \mathrm{~mm}$ (range, 11 to $18 \mathrm{~mm}$ ). Other complications (significant bleeding, infection, vasovagal reaction, contralateral skin piercing) were not observed.

\section{Discussion}

This study reports our early experience with MRIguided VABB. We had no prior experience in MRIguided VABB. Our initial experience showed that the success rate of MRI-guided VABB was $86.7 \%$ (13/15). This rate is slightly lower than the 96 to $100 \%$ technical success rates reported in prior studies of MRI-guided VABB [6-9,11,12,14]. The median procedural time in our study was 55.7 minutes. The median time of MRIguided VABB with the same device in prior studies ranged from 30 to 38 minutes. MRI-guided VABB revealed cancer in four of 13 cases (30.8\%), similar to prior clinical reports of this method. Potential failure of MRIguided VABB were mainly due to the nonvisualization of lesions on the day of biopsy, which may have been caused by changes in the hormonal status or probably

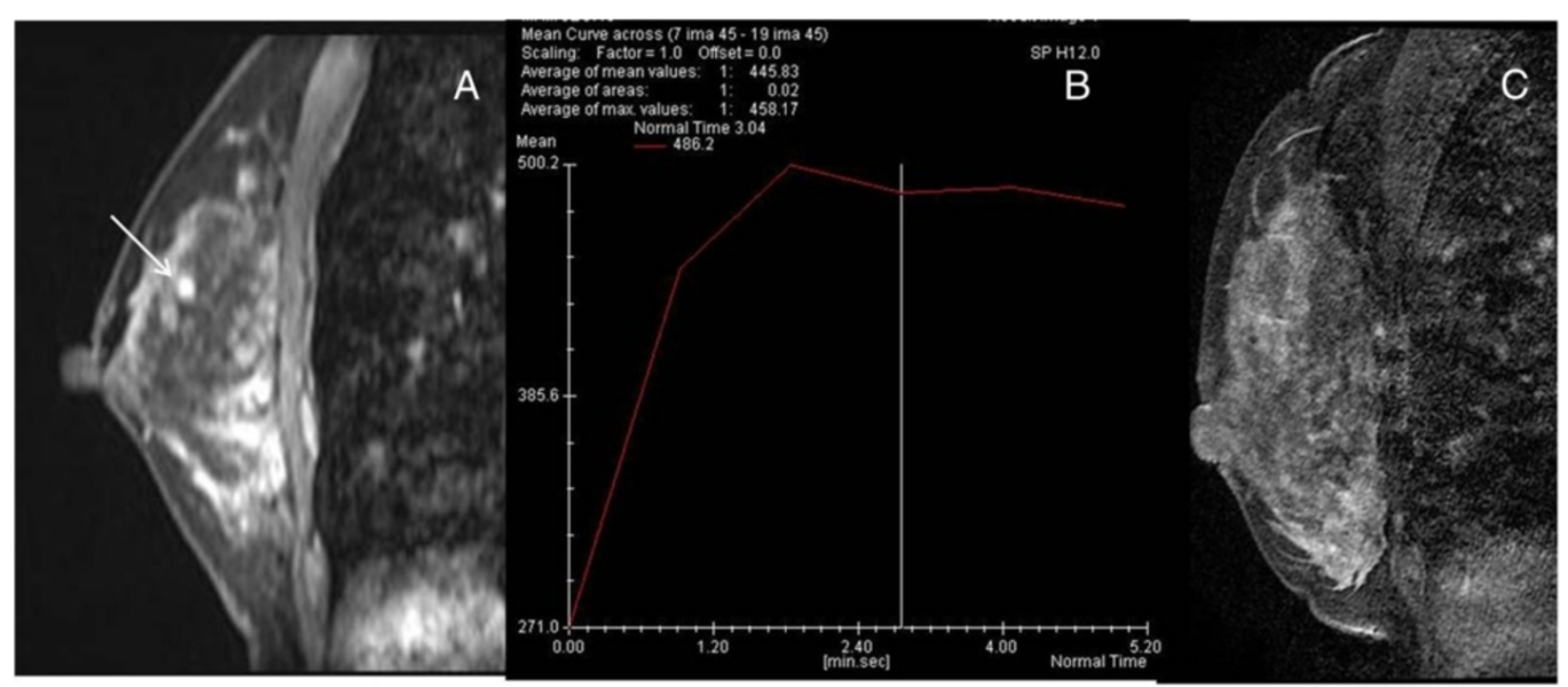

Figure 4 A 38-year-old woman with breast cancer on preoperative magnetic resonance imaging for staging. (A) Sagittal, contrast enhanced T1-weighted image showing oval, smooth, heterogeneous enhancing mass in the right breast, measuring 6 mm, which was categorized into American College of Radiology Breast Imaging Reporting and Data System (BI-RADS) category 4a. (B) Dynamic time-intensity curves of the lesion showing an initial fast uptake followed by a fast washout in the delayed phase (type III), which was categorized into BI-RADS category 4a. (C) Sagittal, contrast enhanced T1-weighted image on the day of biopsy showing no suspicious lesions, and hence magnetic resonance imaging-guided breast biopsy was cancelled. 
due to excessively strong compression of the breast during biopsy. On gaining experience, MRI-guided VABB can be performed in an even shorter amount of time and can have a higher technical success rate than that reported in this study.

Compared with previous reports of studies on MRIguided VABB, our study included a higher proportion of interstitial mammoplasty cases (11 out of 15, 73.3\%), which has not been reported previously (Figure 1, Figure 2 and Figure 3). The malignancy rate in 11 interstitial mammoplasty cases was $36.4 \%$ (4/11), and the mean size of detected cancer was $1.5 \mathrm{~mm}$ (range, 8 to $22 \mathrm{~mm}$ ). Patients who had received silicone or paraffin injection could not be easily or properly evaluated by palpation, mammography, or sonography. As a consequence, breast cancers in patients with interstitial mammoplasty were detected at an advanced stage. Dynamic breast MRI is the modality of choice to detect breast cancer, and MRIguided biopsy is the only method that can confirm suspicious enhancing breast lesions in these patients. According to our previous study [17], the mean diameter of the detected cancers in these patients was $5.5 \mathrm{~cm}$ (range 4.8 to $7.1 \mathrm{~cm})$. In this study, cancers diagnosed by MRI-guided VABB were smaller in size and at an earlier stage compared with those in our previous publication.

The present study has some limitations. The main limitation of this study was the small number of cases. Another limitation was the short period of follow-up in some cases (14 to 35 months) with benign lesions. Thirdly, we did not correlate the hormonal status of the patient and the lesion visibility on MRI. A better approach would be to obtain the patient's menstrual history and usage of hormone replacement therapy before MRI examination.

\section{Conclusions}

MRI-guided VABB is a safe, efficient and effective tool without any major complications in Korean women. During our initial experience a success rate of $86.7 \%$ was achieved, which was quite satisfactory. MRI-guided VABB may contribute to the early diagnosis of breast cancer in interstitial mammoplasty patients in Korea.

\section{Abbreviations}

MRI: Magnetic resonance imaging; VABB: Vacuum-assisted breast biopsy.

\section{Competing interests}

The authors declare that they have no competing interests.

\section{Authors' contributions}

YYA and BJK participated in conception of the study, data collection and analysis, and drafted the manuscript. SHK and JHL participated in data collection and analysis. All authors read and approved the final manuscript.

\section{Acknowledgements}

This study was supported by a research grant from GE Healthcare Medical Diagnostics.

\section{Author details}

'Department of Radiology, St. Vincent's Hospital, College of Medicine, The Catholic University of Korea, $93 \mathrm{Ji}$-Dong, Paldal-Ku, Suwon, Kyunggi-do 442-723, Republic of Korea. ${ }^{2}$ Department of Radiology, Seoul St. Mary's Hospital, College of Medicine, The Catholic University of Korea, 505 BanpoDong, Seocho-Ku, Seoul 137-040, Republic of Korea. ${ }^{3}$ Breast Clinic, Human Medical Imaging and Intervention Center, 12-25 Jamwon-Dong, Seocho-Ku, Seoul 137-902, Republic of Korea.

Received: 12 November 2012 Accepted: 5 August 2013

Published: 16 August 2013

\section{References}

1. Orel SG, Schnall MD, Newman RW, Powell CM, Torosian MH, Rosato EF MR imaging-guided localization and biopsy of breast lesions: initial experience. Radiology 1994, 193:97-102.

2. Gebauer B, Bostanjoglo M, Moesta KT, Schneider W, Schlag PM, Felix R Magnetic resonance-guided biopsy of suspicious breast lesions with a handheld vacuum biopsy device. Acta Radiol 2006, 47:907-913.

3. Ghate SV, Rosen EL, Soo MS, Baker JA: MRI-guided vacuum-assisted breast biopsy with a handheld portable biopsy system. AJR Am J Roentgenol 2006, 186:1733-1736.

4. Han BK, Schnall MD, Orel SG, Rosen M: Outcome of MRI-guided breast biopsy. AJR Am J Roentgenol 2008, 191:1798-1804.

5. Heywang-Kobrunner SH, Heinig A, Pickuth D, Alberich T, Spielmann RP: Interventional MRI of the breast: lesion localisation and biopsy. Eur Radiol 2000, 10:36-45.

6. Heywang-Kobrunner SH, Heinig A, Schaumloffel U, Viehweg P, Buchmann J, Lampe D, Spielmann R: MR-guided percutaneous excisional and incisional biopsy of breast lesions. Eur Radiol 1999, 9:1656-1665.

7. Lehman CD, Deperi ER, Peacock S, McDonough MD, Demartini WB, Shook J: Clinical experience with MRI-guided vacuum-assisted breast biopsy. AJR Am J Roentgenol 2005, 184:1782-1787.

8. Liberman L, Bracero N, Morris E, Thornton C, Dershaw DD: MRI-guided 9-gauge vacuum-assisted breast biopsy: initial clinical experience. AJR Am J Roentgenol 2005, 185:183-193.

9. Liberman L, Morris EA, Dershaw DD, Thornton CM, Van Zee KJ, Tan LK: Fast MRI-guided vacuum-assisted breast biopsy: initial experience. AJR Am J Roentgenol 2003, 181:1283-1293.

10. Orel SG, Rosen M, Mies C, Schnall MD: MR imaging-guided 9-gauge vacuum-assisted core-needle breast biopsy: initial experience. Radiology 2006, 238:54-61.

11. Perlet C, Heinig A, Prat X, Casselman J, Baath L, Sittek H, Stets C, Lamarque J, Anderson I, Schneider P, Taourel P, Reiser M, Heywang-Köbrunner SH: Multicenter study for the evaluation of a dedicated biopsy device for MR-guided vacuum biopsy of the breast. Eur Radiol 2002, 12:1463-1470.

12. Perlet C, Heywang-Kobrunner SH, Heinig A, Sittek H, Casselman J, Anderson I, Taourel P: Magnetic resonance-guided, vacuum-assisted breast biopsy: results from a European multicenter study of 538 lesions. Cancer 2006, 106:982-990.

13. Tozaki M, Yamashiro N, Fukuma E: MR-guided vacuum-assisted breast biopsy using a non-titanium needle. Magn Reson Med Sci 2007, 6:259-264.

14. Tozaki M, Yamashiro N, Sakamoto M, Sakamoto N, Mizuuchi N, Fukuma E: Magnetic resonance-guided vacuum-assisted breast biopsy: results in 100 Japanese women. Jpn J Radiol 2010, 28:527-533.

15. Tozaki M, Yamashiro N, Suzuki T, Kawano N, Ozaki S, Sakamoto N, Abe S, Ogawa T, Katayama N, Tsunoda Y, Fukuma E: MR-guided vacuum-assisted breast biopsy: is it an essential technique? Breast Cancer 2009, 16:121-125.

16. American College of Radiology: Breast Imaging Reporting and Data System, Breast Imaging Atlas. 4th edition. Reston, Va, USA: American College of Radiology; 2003.

17. Kang BJ, Kim SH, Choi JJ, Lee JH, Cha ES, Kim HS, Park CS, Whang IY: The clinical and imaging characteristics of breast cancers in patients with interstitial mammoplasty. Arch Gynecol Obstetr 2010, 281:1029-1035.

doi:10.1186/1477-7819-11-200

Cite this article as: An et al:: Usefulness of magnetic resonance imagingguided vacuum-assisted breast biopsy in Korean women: a pilot study. World Journal of Surgical Oncology 2013 11:200. 